\title{
Preliminary Studies Of A Phase Modulation Technique For Measuring Chromaticity
}

\author{
Cheng-Yang Tan ${ }^{1,2}$ \\ Fermi National Accelerator Laboratory \\ Accelerator Division/Tevatron, MS341 \\ P.O. Box 500 \\ Batavia, IL 60510-0500
}

\begin{abstract}
The classical method for measuring chromaticity is to slowly modulate the RF frequency and then measuring the betatron tune excursion. The technique that is discussed in this paper modulates instead the phase of the RF and then the chromaticity is obtained by phase demodulating the betatron tune. However, this technique requires knowledge of the betatron frequency in real time in order for the phase to be demodulated. Fortunately, the Tevatron has a tune tracker based on the phase locked loop principle which fits this requirement. A preliminary study with this technique has showed that it is a promising method for doing continuous chromaticity measurement and raises the possibility of doing successful chromaticity feedback with it.
\end{abstract}

Keywords: chromaticity, phase modulation.

PACS: $29.27 \mathrm{Fh}, 29.27 .-\mathrm{a}$

\section{INTRODUCTION}

Tracking the chromaticity especially during snapback is critical for the Large Hadron Collider (LHC). Various methods have been proposed to do this: the Bruening method, the head-tail method etc. In this paper, we will discuss the method by D. McGinnis.[1] His idea is to phase modulate the beam with the accelerating RF and then detect this phase modulation with a transverse pickup. By phase demodulating this transverse signal, the chromaticity can be obtained. Although he had demonstrated that the chromaticity can be measured, he faced the problem that the betatron tune would drift over time and the phase demodulation circuit (which is a vector signal analyzer used in phase demodulation mode) would lose lock and thus would be unable to report the chromaticity. It was not until the Tevatron tune tracker (TT) was declared operational, $[3,4]$ that it became clear that the McGinnis method could be made to work. First, the betatron tune drift problem would be solved because the TT would always be locked to the betatron tune (or to a synchrotron sideband of the betatron tune). Second the phase modulation would be chosen so that it would be outside the closed loop bandwidth of the TT and thus the TT would not "see" the modulation and respond to it.

${ }^{1}$ Funded by Universities Research Association Inc. under Contract No. DE-AC02-76CH03000 with the United States Department of Energy.

${ }^{2}$ Email address: cytan@fnal.gov 


\section{THEORY}

The McGinnis method $[1,2]$ starts with the modulation of the RF frequency $\dot{\phi}_{\mathrm{RF}}(t)$ with a sinusoid

$$
\dot{\phi}_{\mathrm{RF}}(t)=\omega_{\mathrm{RF}}+\Delta \phi_{\text {mod }} \times \Omega_{\text {mod }} \cos \left(\Omega_{\text {mod }} t\right)
$$

where $\omega_{\mathrm{RF}}$ is the RF frequency when there is no modulation, $\Delta \phi_{\bmod }$ is the amplitude of the phase modulation and $\Omega_{\bmod }$ is the RF phase modulation. He showed that the betatron phase $\phi_{\beta}$ will then be modulated and its amplitude is linearly dependent on the chromaticity $\chi$. The relationship between $\phi_{\beta}$ to $\chi$ was calculated by him to be

$$
\phi_{\beta}(t)=\omega_{\mathrm{rev}} Q_{0} t+\frac{\Delta \phi_{\mathrm{mod}}}{h}\left(Q_{0}-\frac{\chi}{\eta}\right) \sin \Omega_{\mathrm{mod}} t+\Delta \phi_{\mathrm{s}}\left(Q_{0}-\frac{\chi}{\eta}\right) \sin \left(\Omega_{s} t+\theta_{s}\right)+\phi_{\beta_{0}}
$$

where $\omega_{\text {rev }}$ is the revolution frequency, $Q_{0}$ is the betatron tune, $h$ is the harmonic number, $\eta$ is the slip factor, $\Delta \phi_{\mathrm{s}}$ is the amplitude of the synchrotron phase modulation, $\Omega_{s}$ is the synchrotron frequency, $\theta_{s}$ is the synchrotron phase and $\phi_{\beta_{0}}$ is the betatron phase at $t=0$.

In reality, we measure the betatron tune by taking the difference of the image current $I_{\Delta}$ between two plates of a pickup. This difference is

$$
\begin{aligned}
I_{\Delta}(t)= & \omega_{\mathrm{rev}} q_{b} \frac{A}{\sqrt{\varepsilon}} \cos \left[\phi_{\beta}(t)\right] \sum_{n=-\infty}^{\infty} \delta\left(\phi_{r}(t)-2 n \pi-\phi_{r}(0)\right) \\
= & \frac{\omega_{\mathrm{rev}} q_{b}}{2} \frac{A}{\sqrt{\varepsilon}} \sum_{k=0}^{\infty} C_{k} \sum_{m=-\infty}^{\infty} \sum_{n=-\infty}^{\infty} J_{m}\left(Y_{+}\right) J_{n}\left(Z_{+}\right) \cos \left(\omega_{k m n}^{+} t+\psi_{+}\right) \\
& +\frac{\omega_{\mathrm{rev}} q_{b}}{2} \frac{A}{\sqrt{\varepsilon}} \sum_{k=0}^{\infty} C_{k} \sum_{m=-\infty}^{\infty} \sum_{n=-\infty}^{\infty} J_{m}\left(Y_{-}\right) J_{n}\left(Z_{-}\right) \cos \left(\omega_{k m n}^{-} t+\psi_{-}\right)
\end{aligned}
$$

where

$$
\phi_{r}(t)=\omega_{\mathrm{rev}} t+\frac{\Delta \phi_{\mathrm{mod}}}{h} \sin \Omega_{\mathrm{mod}} t+\Delta \phi_{\mathrm{s}} \sin \left(\Omega_{s} t+\theta_{s}\right)+\phi_{\beta_{0}}
$$

and $q_{b}$ is the charge of the bunch, $A$ is the betatron amplitude at the pickup,

$C_{0}=1 / 2 \pi, C_{k}=1 / \pi, Y_{ \pm}=\left(k \mp Q_{0} \pm \frac{\chi}{\eta}\right) \Delta \phi_{s}, Z_{ \pm}=\left(k \pm Q_{0} \mp \frac{\chi}{\eta}\right) \frac{\Delta \phi_{\bmod }}{h}$,

$\psi_{ \pm}=k \phi_{r}(0) \pm \phi_{\beta}(0), \omega_{k m n}^{ \pm}=\left(k \pm Q_{0}\right) \omega_{r e v}+m \Omega_{s}+n \Omega_{\text {mod }}$, and $\varepsilon$ is the transverse

emittance.

Therefore, (4) shows that at a given betatron mode $k$, there are an infinite number of modes $m$ associated with the synchrotron frequency. Around any mode $(k, m)$ there are an infinite number of modes $n$ associated with the RF modulation. 
In general, the TT will just lock to one synchrotron sideband. We can still obtain the chromaticity by phase demodulating this sideband by using it as the carrier frequency. Suppose the synchrotron line that the TT locks to is mode $(k, m,+)$ then its mode frequency is $\omega_{k m}^{+}=\left(k+Q_{0}\right) \omega_{\text {rev }}+m \Omega_{s}$. The spectrum of modes associated with the RF modulation in temporal space is

$$
\begin{aligned}
I_{\Delta}(t ; k, m, n,+) & \propto \sum_{n=-\infty}^{\infty} J_{n}\left(Z_{+}\right) \cos \left(\omega_{k m}^{+} t+n \Omega_{\bmod } t+\psi_{+}\right) \\
& =\cos \left(\omega_{k m}^{+} t+Z_{+} \sin \Omega_{\text {mod }} t+\psi_{+}\right)
\end{aligned}
$$

By using the well known formula

$$
e^{i Z \sin \theta}=\sum_{n=-\infty}^{\infty} J_{n}(Z) e^{i n \theta}
$$

This result shows that the carrier frequency is $\omega_{k m}^{+}$and the phase is modulated by $Z_{+} \sin \Omega_{\bmod } t$. More importantly, it also shows that we can phase demodulate using any synchrotron line as the carrier to obtain $\chi$ because the peak amplitude of the modulation $Z_{+}$is both independent of $m$ and the amplitude of the synchrotron line. A similar argument will show that this is also true for the synchrotron line $(k, m,-)$ where $Z_{-}$is the peak of the modulation amplitude.

\section{Sign of the Chromaticity}

It is obvious that when we just apply phase demodulation, we can only measure $\left|Z_{ \pm}\right|$and clearly this is insufficient to determine the sign of the chromaticity. To know the sign, we must transmit the modulation frequency from the source which is close to the RF cavities to the location of the TT and the chromaticity tracker (CT). Once this modulation frequency is transmitted to the location of the CT, we can multiply it with the demodulated signal from the CT, i.e.

$$
W_{ \pm}=\left(Z_{ \pm} \sin \Omega_{\text {mod }} t\right) \times B \sin \left(\Omega_{\text {mod }} t+\theta\right)
$$

where $B>0$ is the amplitude and $\theta$ is the phase of the modulation frequency. $\theta$ is measured at the CT location w.r.t. the modulation frequency at the source. Note that if $\Omega_{\text {mod }} \approx(2 \pi \times 23) \mathrm{s}^{-1}$, then its wavelength is approximately $10^{7} \mathrm{~m}$, this means that despite the fact that the modulation source and the CT are at two different locations, $\theta \approx 0$. Thus

$$
W_{ \pm}=\frac{B Z_{ \pm}}{2}\left(1-\cos 2 \Omega_{\text {mod }} t\right)
$$

We only want the DC term, $W_{D C}=B Z_{ \pm} / 2$

$$
\frac{2 W_{D C}}{B}=\left(k-Q_{0}+\frac{\chi}{\eta}\right) \frac{\Delta \phi_{\mathrm{mod}}}{h}
$$


if we only consider the $Z_{-}$term. And to get the sign of the chromaticity, we need to do a bit of processing to get

$$
\frac{2 W_{D C}}{B}-\left(k-Q_{0}\right) \frac{\Delta \phi_{\mathrm{mod}}}{h}=\frac{\chi}{\eta} \times \frac{\Delta \phi_{\mathrm{mod}}}{h}
$$

Therefore, not only the sign of the chromaticity but the chromaticity itself can be obtained from here.

\section{Example}

For the Tevatron, if we use the standard 21.4 MHz Schottky detector, we measure the betatron tune from the lower sideband for $k=449$. Since the Tevatron betatron tunes are between $0.5 \omega_{\text {rev }}$ and $0.6 \omega_{\text {rev }}$, this means that $k-Q_{0} \approx 448.5$. The peak of the phase demodulation $\left|Z_{-}\right|$is related to the chromaticity $\chi$ by the formula

$$
\begin{aligned}
|\chi| & =\eta\left|\frac{\sqrt{2}\left|Z_{-}\right| h}{\Delta \phi_{\text {mod }}}-\left(k-Q_{0}\right)\right| \\
& = \begin{cases}0.0029|9018.4| Z_{-}|-448.5| & \text { if } \Delta \phi_{\bmod }=10^{\circ} \\
0.0029|18036.8| Z_{-}|-448.5| & \text { if } \Delta \phi_{\bmod }=5^{\circ}\end{cases}
\end{aligned}
$$

if $\left|Z_{-}\right|$is measured in units of $\mathrm{rad} \bullet \mathrm{rms}$ (from a vector signal analyzer) $h=1113$, and $\eta=0.0029$.

\section{Limitations}

There are two possible limitations for the McGinnis method. The first, from (2), shows that this method would fail at transition because $\eta=0$. The second is whether the longitudinal dampers will damp out the phase modulation. We can think of the slow phase modulation on the beam as a mode 0 coupled bunch mode. The Tevatron longitudinal damper does not damp mode 0 at this time[5], but instead relies on the RF frequency being set to the correct side of the revolution harmonic to keep the beam stable, i.e. using the Robinson stability criterion. Therefore, in principle, the longitudinal dampers should not affect this method.

\section{MEASUREMENTS}

All these measurements were performed by phase modulating the Tevatron RF at $\Omega_{\text {mod }}=(2 \pi \times 23) \mathrm{s}^{-1}$. The TT was used to lock to the betatron tune. Because the betatron tune was excited with a clear carrier from the TT, a vector signal analyzer (VSA) could then be used to demodulate the betatron tune. Unfortunately, for this setup we could not just type in the TT excitation frequency into the VSA and use zero span because the betatron tune drifts and so we must rely on the VSA's phase lock loop to find the carrier in a non-zero bandwidth. In these experiments, the VSA was 
set to a bandwidth of $200 \mathrm{~Hz}$ (and thus a $100 \mathrm{~Hz}$ span display of the phase demodulation) centred on the betatron tune. However, in this mode of operation, the VSA update rate was quite slow $\sim 2$ to 3 seconds because it needed steady state to be reached. The setup is shown in Figure 1.
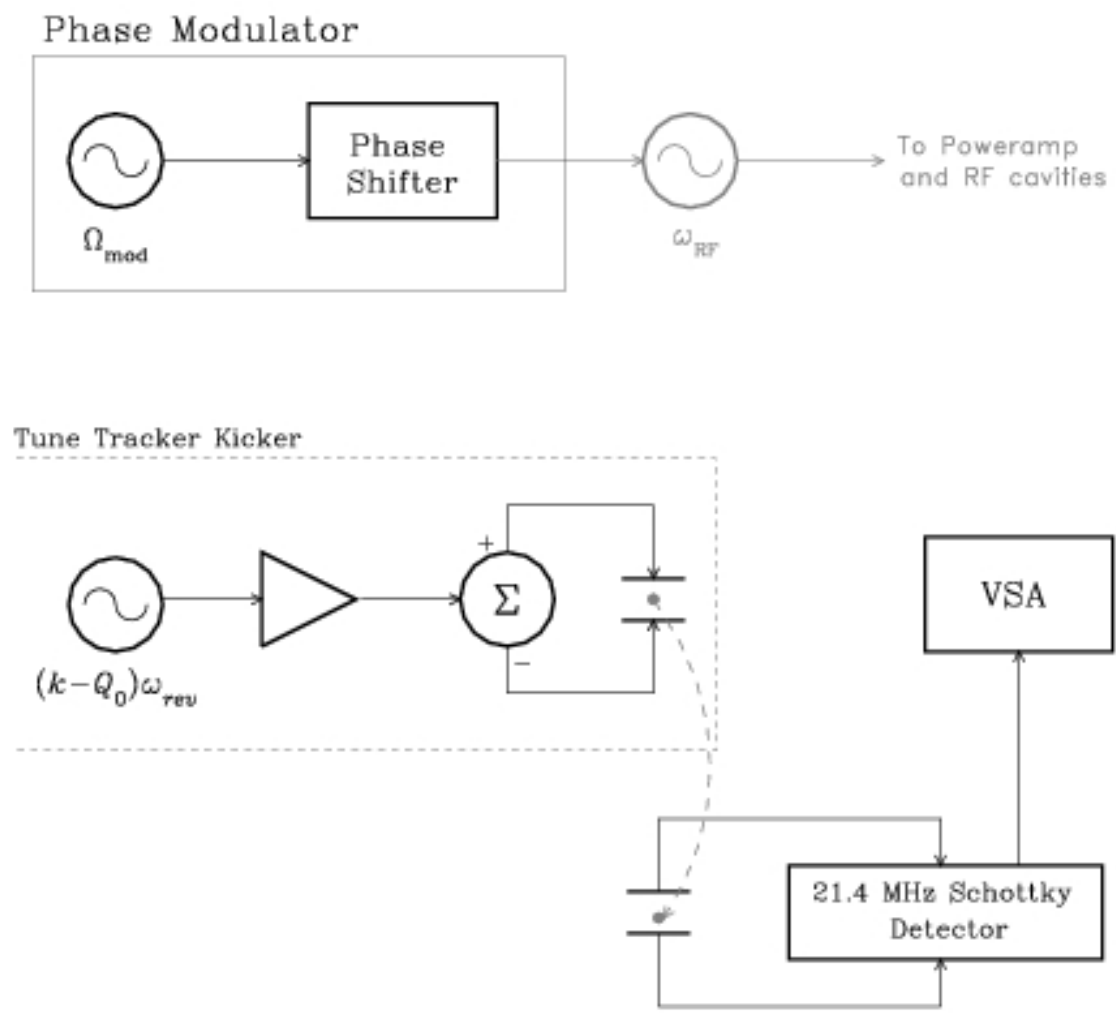

FIGURE 1. The setup for these measurements starts with modulating the beam with the RF. The TT is locked to the betatron tune and its excitation frequency serves as the carrier for the VSA to phase demodulate the betatron tune.

Two sets of data were taken: $\Delta \phi_{\bmod }=10^{\circ}$ and $5^{\circ}$. The result of demodulating the betatron tune by the VSA is shown in Figure 3. Clearly, from here, the height of the $23 \mathrm{~Hz}$ peak for the $\Delta \phi_{\text {mod }}=10^{\circ}$ case is approximately twice that of the $\Delta \phi_{\text {mod }}=5^{\circ}$ case for the same chromaticity like we would expect from the $Z_{-}$formula.

We varied the chromaticity $\chi$ and measured $\left|Z_{-}\right|$from the VSA. We then used the formula from (11) to calculate $\chi$ from $\left|Z_{-}\right|$. The fit between the expected chromaticity $\chi_{\text {expected }}$ and the chromaticity from McGinnis' method $\chi_{\text {McGinnis }}$ for the two cases $\Delta \phi_{\text {mod }}=10^{\circ}$ and $5^{\circ}$ are shown in Figure 2 . The result of the fits were $\chi_{\text {McGinnis }}$

$$
\chi_{\text {McGinnis }}=(0.83 \pm 0.05) \chi_{\text {expected }}-(0.6 \pm 0.2)
$$

if $\Delta \phi_{\text {mod }}=10^{\circ}$ and 


$$
\chi_{\text {McGinnis }}=(1.0 \pm 0.1) \chi_{\text {expected }}-(1.7 \pm 0.5)
$$

if $\Delta \phi_{\text {mod }}=5^{\circ}$. Note that the sign of the chromaticity, which cannot be determined directly from the height of the phase demodulation with the VSA, is assumed to be the same as that of $\chi_{\text {expected }}$.

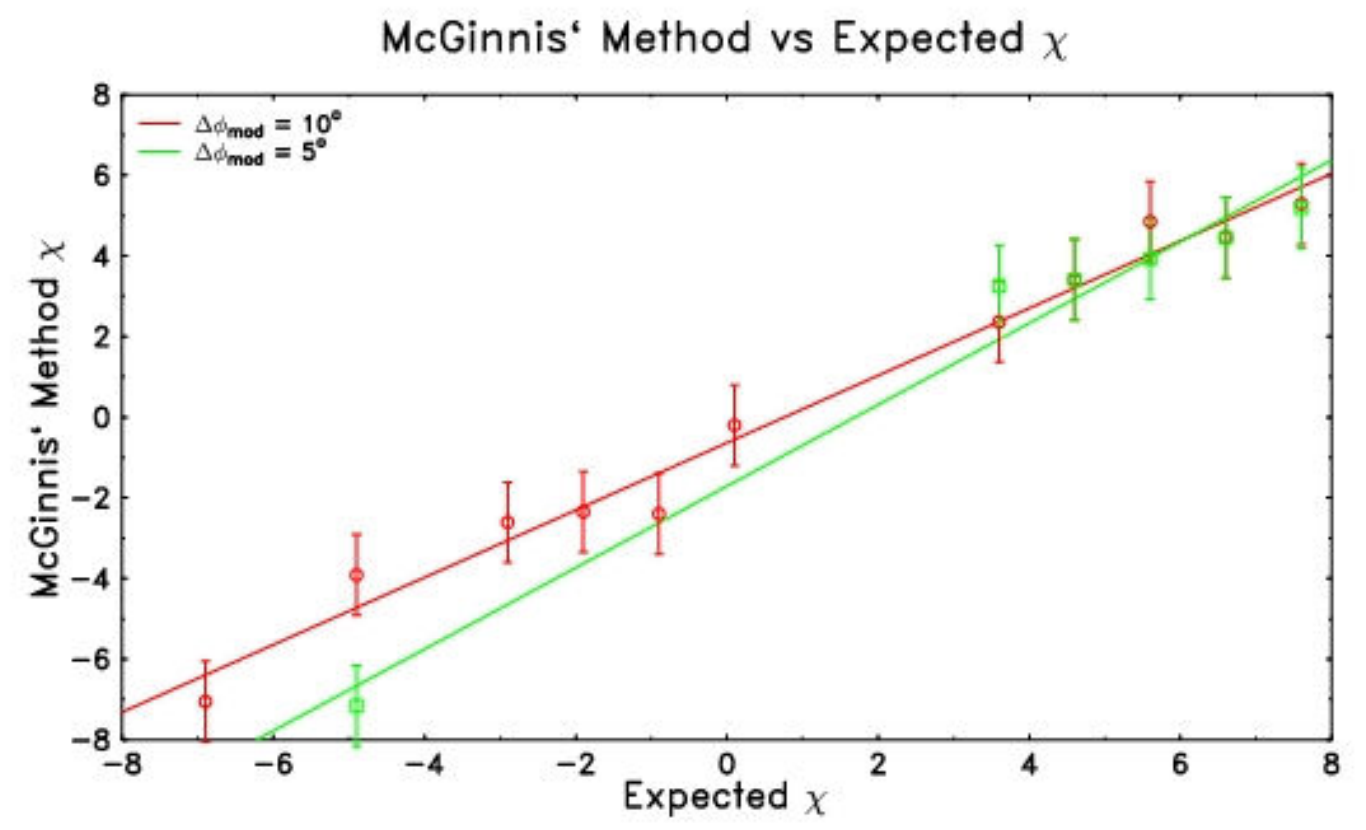

FIGURE 2. Comparing the McGinnis' method to the expected chromaticity for $\Delta \phi_{\bmod }=10^{\circ}$ and $5^{\circ}$.

\section{Other Measurements}

One of the things that we need to show is that the TT will ignore the phase modulation. From Figure 4, the phase modulation is turned on with $\Delta \phi_{\bmod }=5^{\circ}$, $\Omega_{\text {mod }}=(2 \pi \times 23) \mathrm{s}^{-1}$ and the TT is on and tracking. Clearly, we see that the TT tracks the tune changes when the chromaticity knob (the tune change is a side effect of changing the chromaticity with the chromaticity knob) is changed. As expected, the TT does not track the phase modulation because $\Omega_{\text {mod }}$ is outside the closed loop bandwidth of the TT. 


\section{Date: 01-14-06 Time: 03:17 AM}

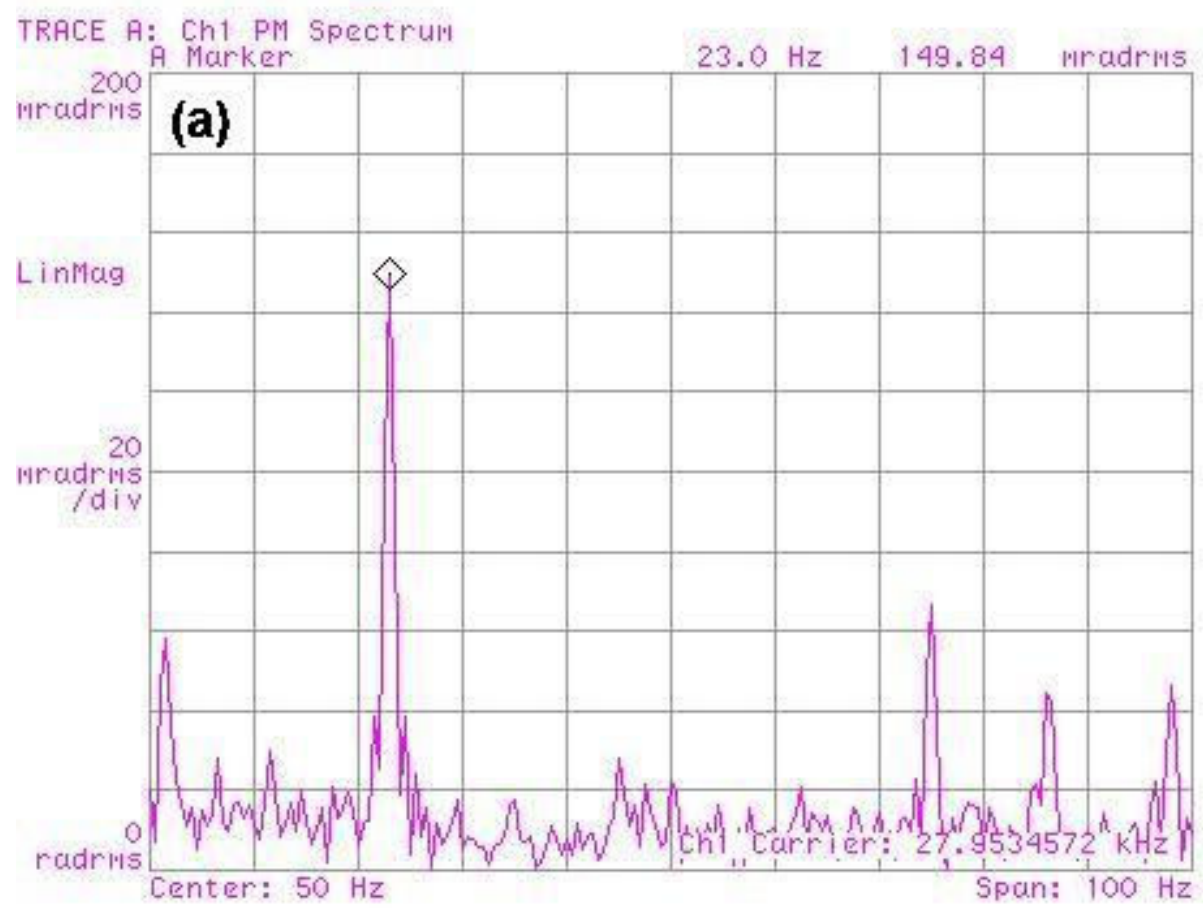

\section{Date: 01-14-06 Time: 03:18 AM}

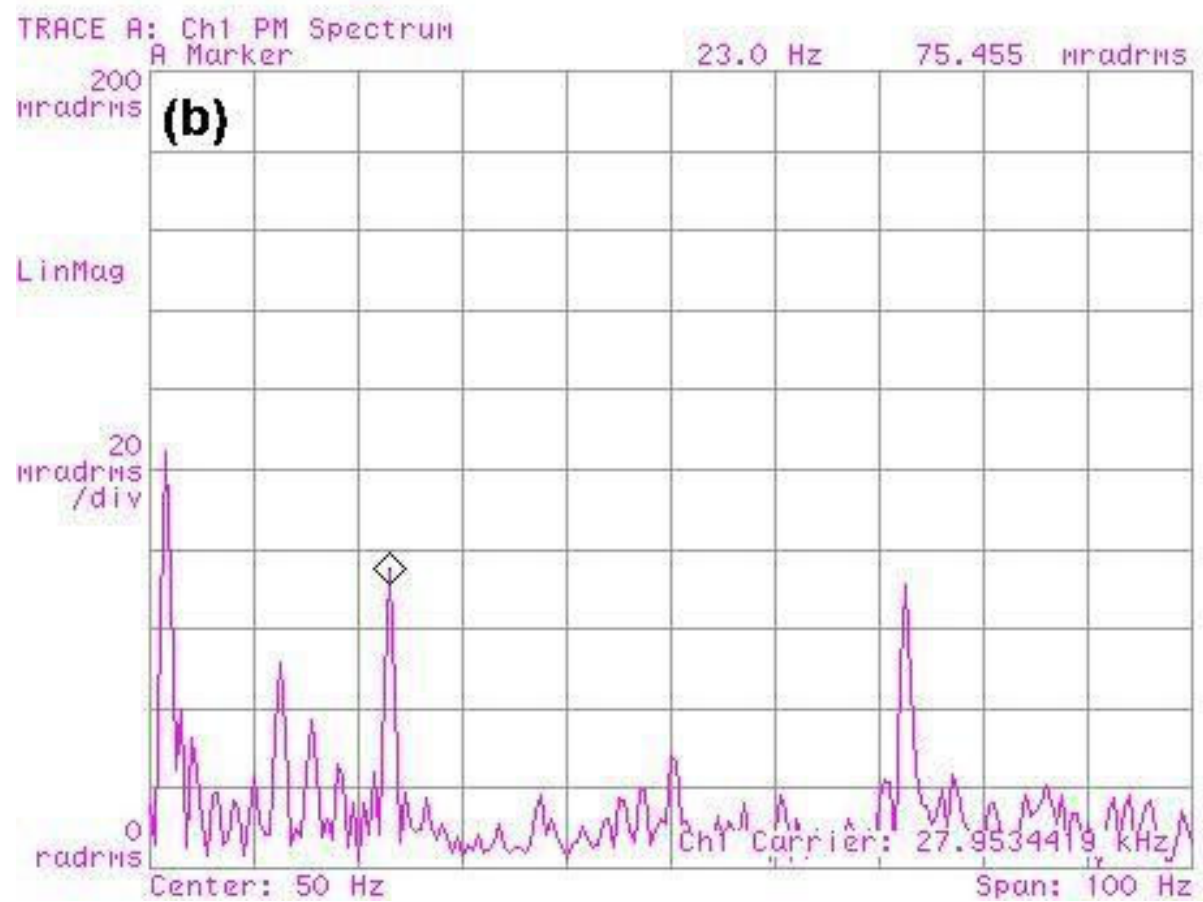

FIGURE 3. These figures show the result of demodulating the betatron tune. (a) shows the case when $\Delta \phi_{\text {mod }}=10^{\circ}$ and (b) shows the case when $\Delta \phi_{\text {mod }}=5^{\circ}$. Clearly the height of the $23 \mathrm{~Hz}$ peak of (a) is approximately twice that of (b) like we expect for the same chromaticity. 


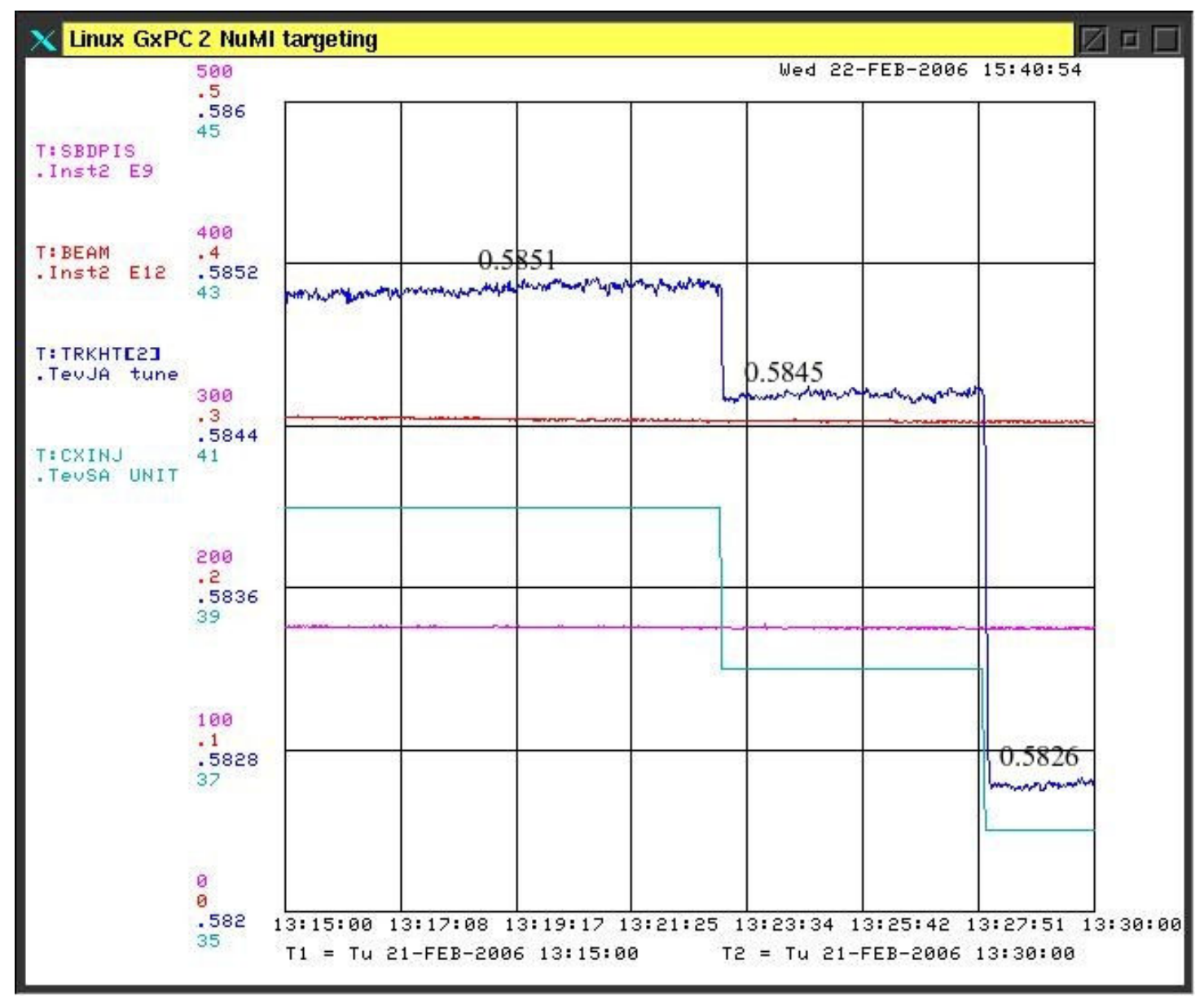

FIGURE 2. Tracking with $\Delta \phi_{\text {mod }}=5^{\circ}$. The TT parameter T:TRKHT[2] tracks the tune changes when the chromaticity knob is changed T:CXINJ. The bunched beam T:SBDPIS and the total current $\mathrm{T}$ :BEAM remains constant during this measurement.

\section{CONCLUSION}

We have performed a preliminary study of the McGinnis' method and have found that the TT does ignore phase modulations outside its closed loop bandwidth while locked to the betatron tune. We have also verified that a $\Omega_{\bmod }=(2 \pi \times 23) \mathrm{s}^{-1}$, $\Delta \phi_{\text {mod }}=5^{\circ}$ phase modulation does not do any harm to the beam and is sufficient for the VSA to measure it. The studies with the $21.4 \mathrm{MHz}$ Schottky have shown that the chromaticity measured with McGinnis' method is close to what we expect from measuring the chromaticity at one point with the RF change method (See Introduction) and then extrapolating it with a "calibrated" knob. With the qualified success of the McGinnis' method, we have proposed and discussed a design of a CT. The electronics for this design is being drawn up and built at this time. 


\section{ACKNOWLEDGMENTS}

The author wishes to thank

1. The Tevatron group for supporting these studies.

2. US-LARP for funding part of these studies.

\section{REFERENCES}

1. D. McGinnis, "Chromaticity Measurements using Phase Modulated RF and Vector Signal Analyzers", PBAR Note 656, Fermilab, 2001.

2. C.Y. Tan, "Preliminary Studies of a Chromaticity Tracker", Fermilab Technical Memo, TM-2346, 2006.

3. C.Y. Tan, "The Tevatron Tune Tracker PLL --- Theory, Implementation and Measurements", TM-2275, Fermilab, 2004.

4. C.Y. Tan, "Tune Tracking with a PLL in the Tevatron", TM-2275, Nucl. Instrum. Meth. A 557,615-202 (2006).

5. C.Y. Tan and J. Steimel, "The Tevatron Longitudinal Dampers", TM-2184, Fermilab, 2002. 